RESEARCH ARTICLE

\title{
Social touch increases dominant gaze in social confrontations
}

\author{
Isabell M. Meier ${ }^{1}$, Jack van Honk ${ }^{1,2,3}$, Peter A. Bos ${ }^{4}$, David Terburg ${ }^{1,2}$ \\ ${ }^{1}$ Department of Experimental Psychology, Utrecht University, Utrecht, The Netherlands \\ ${ }^{2}$ Department of Psychiatry and Mental Health, Groote Schuur Hospital, MRC Unit on Anxiety \& Stress Disorders, \\ University of Cape Town, Cape Town, South Africa \\ ${ }^{3}$ Institute of infectious Diseases and Molecular Medicine, University of Cape Town, South Africa \\ ${ }^{4}$ Institute of Education and Child studies, Leiden University, The Netherlands
}

*Author for correspondence (Isabell.m.meier@gmail.com); Department of Experimental Psychology, Utrecht University, Heidelberglaan 1, 3584 CS Utrecht, The Netherlands. Phone: +31 (0)30 2534368

Running Head: Social touch alters gaze behavior 
Social touch alters gaze behavior $\mid 2$

\begin{abstract}
Positive effects of touch have been shown on the psychological and physiological level, with touch for example protecting us against the negative consequences of stress. Social touch however also induces feelings of security and willingness to take risks, characteristics of socially dominant behavior. The current study ( $\mathrm{N}=24$, female) investigates the effect of social touch on speed of gaze-aversion from eye-contact, an implicit marker of dominance-submissive behavior. Using interactive eye-tracking we show that social touch slows down gaze-aversion from subliminally presented angry compared to happy eye-contact. Recently, we reported slower gaze-aversion from subliminally presented facial anger in subjects with high levels of social dominance and low levels of social anxiety. The present results thus provide evidence that social touch induces gaze behaviors characteristic for high social dominance and low social anxiety. By slowing down gazeaversion to facial anger social touch promotes the implicit-reactive behavioral tendency to engage in social confrontation.
\end{abstract}

Keywords: Social touch, Gaze-aversion, Social dominance, Eye-tracking 
Social touch alters gaze behavior $\mid 3$

\section{Introduction}

Social touch is crucial in our day-to-day lives. It is used to communicate emotions, contributes to resilience resources, and creates and strengthens social bonds (Burleson \& Davis, 2014; Dunbar, 2010; Morrison, Löken, \& Olausson, 2010). Social touch protects from and speeds up recovery after stress which is argued to provide for a sense of social security in humans (Jakubiak \& Feeney, 2016b, 2016a) and other primates (Dunbar, 2010). Indeed, social touch attenuated attentional bias to social threat in children (Brummelman, Terburg, Smit, Bögels, \& Bos, 2018), and initiated processes of emotion regulation resulting in a reduction of threat-related brain activity (Coan, Schaefer, \& Davidson, 2006). Psychologically, even imagined social touch interactions increase the likelihood to engage more confidently in a social confrontation and promote exploration (Jakubiak \& Feeney, 2016b). Interesting in this respect is that social touch acts on the core of our stress-system, decreasing heart-rate and cortisol responses to social stress in humans (Ditzen et al., 2007; Morrison, 2016), and inducing endorphin release in primates (Dunbar, 2010). These physiological findings suggest that social touch has the potential to not only promote social confrontation through psychological mechanisms (Jakubiak \& Feeney, 2016b), but will do so implicitly by affecting automatic behavioral tendencies. Here, we therefore hypothesize that social touch will promote the implicit behavioral tendency to engage in social confrontation.

Behavioral mechanisms of dominance and submission are crucial to establish and maintain social hierarchy. In non-human primates as well as in humans, staring back at an angry individual is a confrontation for social dominance (Mazur \& Booth, 1998). Averting the gaze from threatening eye-contact, on the other hand, is an indicator of subordinate behavior serving to prevent direct or physical confrontation and is linked to high social anxiety traits (Terburg, Hooiveld, Aarts, Kenemans, \& van Honk, 2011; Terburg et al., 2016).

In the current study we use a validated gaze-aversion paradigm to measure the effect of touch on dominant behavior in the context of social confrontation. Using an interactive eye tracking approach, we measure the time it takes participants to actively avert their gaze from eye-contact with angry, happy and neutral faces. Specifically the contrast of eye-contact with angry compared to happy faces has previously been associated with social dominance motives (Enter, Terburg, Harrewijn, Spinhoven, \& Roelofs, 2016; Terburg, Aarts, \& Honk, 2012; Terburg et al., 2011, 2016). Slower gaze-aversion from angry compared to happy faces thereby reflects social dominance and reduced socially anxious behavior, whereas an increased gaze-aversion speed is related to submissive traits and social anxiety. In addition, socially anxious individuals show a shift from fast to slower gaze-avoidance from angry compared to happy faces after an administration of testosterone. Important in this context, the emotional expressions are presented subliminally which ensures that the gaze-aversion task measures social dominance on an implicit and reactive level (Terburg et al., 2016). With social touch increasing resilience and reducing behavioral tendencies related to social anxiety (Brummelman et al., 2018; Burleson \& Davis, 2014; Jakubiak \& Feeney, 2016a, 2016b), we expect that social touch increases social dominance as indexed by slower gaze-aversion from unseen angry compared to happy eye-contact. 
Social touch alters gaze behavior $\mid 4$

\section{Methods}

\section{Participants}

Thirty-two healthy female volunteers participated in the study (age: $\mathrm{m}=20.9, \mathrm{SD}=2.3$ ). With the here reported task being part of a larger study, the sample size was determined based on previous studies using a comparable range of tasks and measures (D. Hofman, Bos, Schutter, \& van Honk, 2012; Dennis Hofman, Terburg, van Wielink, \& Schutter, 2013; Terburg et al., 2012). Further we conducted a power analysis with medium to large effect size of $\mathrm{f}=.35$ (cf. D. Hofman et al., 2012; Dennis Hofman et al., 2013; Terburg, Aarts, \& Honk, 2012) for a within-subject repeated measures ANOVA, carried out with G*Power 3 (Erdfelder, 2007; Erdfelder \& Buchner, 1996). We chose to include female participants only to avoid sex differences as a confound regarding the touch manipulation, which was done by a female experimenter. All participants included in the study used oral contraceptives, which ensures that hormonal fluctuations do not influence the study results. In total 8 participants were excluded from analysis, resulting in $\mathrm{N}=24$ for the analysis. One participant was excluded due to technical difficulties, one did not attend the second testing session, five participants scored 15 or higher on the emotion awareness check concerning the task stimuli that was conducted after the experiment (details under the paragraph 'Emotion Awareness Check'), and one participant for whom the experiment had to be recalibrated more than 3SD from the mean repositioning rate $(\mathrm{m}=4.3, \mathrm{SD}=13.8$; defined as amount of times the eye-tracker checks the position of the participant in relation to stimulus presentation). Participants received either monetary rewards or course credits. The study was approved by the ethical committee of the faculty of social sciences of Utrecht University.

\section{Gaze-aversion task}

The study was set up as a within-subject cross-over design, each participant attended in total three sessions as part of a larger study. The first session was aimed to be informative on the procedural aspects of the study, it further included several questionnaires, and a measurement of pain threshold/ tolerance which was separated from the experimental sessions to avoid interference with the different tasks. The gaze-aversion task was performed in session two and three, once with prior touch manipulation and once without. Whilst electroencephalography (EEG) and electromyography (EMG) electrodes were placed at the start of session two and three, the here reported task was designed for eye-tracking specifically and does not allow to report EMG or EEG data due to the fast timing of events. The starting order of sessions (touch vs. no-touch) was counterbalanced in random fashion over participants.

The gaze-aversion task started immediately after the touch manipulation, once the experimenter left the room. In the gaze-aversion task three types of emotional stimuli were presented subliminally, displaying happy, angry and neutral facial expressions by 5 male and 5 female actors. A trial developed, starting with a gray pre-mask stimulus with a fixation point at center, followed by a face stimulus displayed in blue, green or red for $27 \mathrm{~ms}$ and then immediately replaced by a 
post-mask stimulus presented in the same color as the face stimulus (see Fig. 1). To assure that masks and stimuli had similar luminance properties, masks were created by cutting-up and randomly reassembling the facial stimuli. During pre-mask display, participants were asked to fixate on the fixation cross, which marked the location of the screen where the eyes of the facial stimulus would appear. The participants gaze was monitored by an eye-tracker to ascertain consistent fixation in the randomly pre-determined time interval $(1000,1500 \mathrm{~ms})$ before stimulus presentation. Below the stimulus area three gray same size circles were displayed at equal distance from the fixation point, each of them changing color (blue, green and red at random location) together with post-mask presentation. The participants task was, as soon as the stimulus turned from gray to color, to identify and look as fast as possible from the fixation point to the circle with the corresponding color. The post-mask disappears once the gaze is shifted to the correct corresponding color and therewith ends the trial.

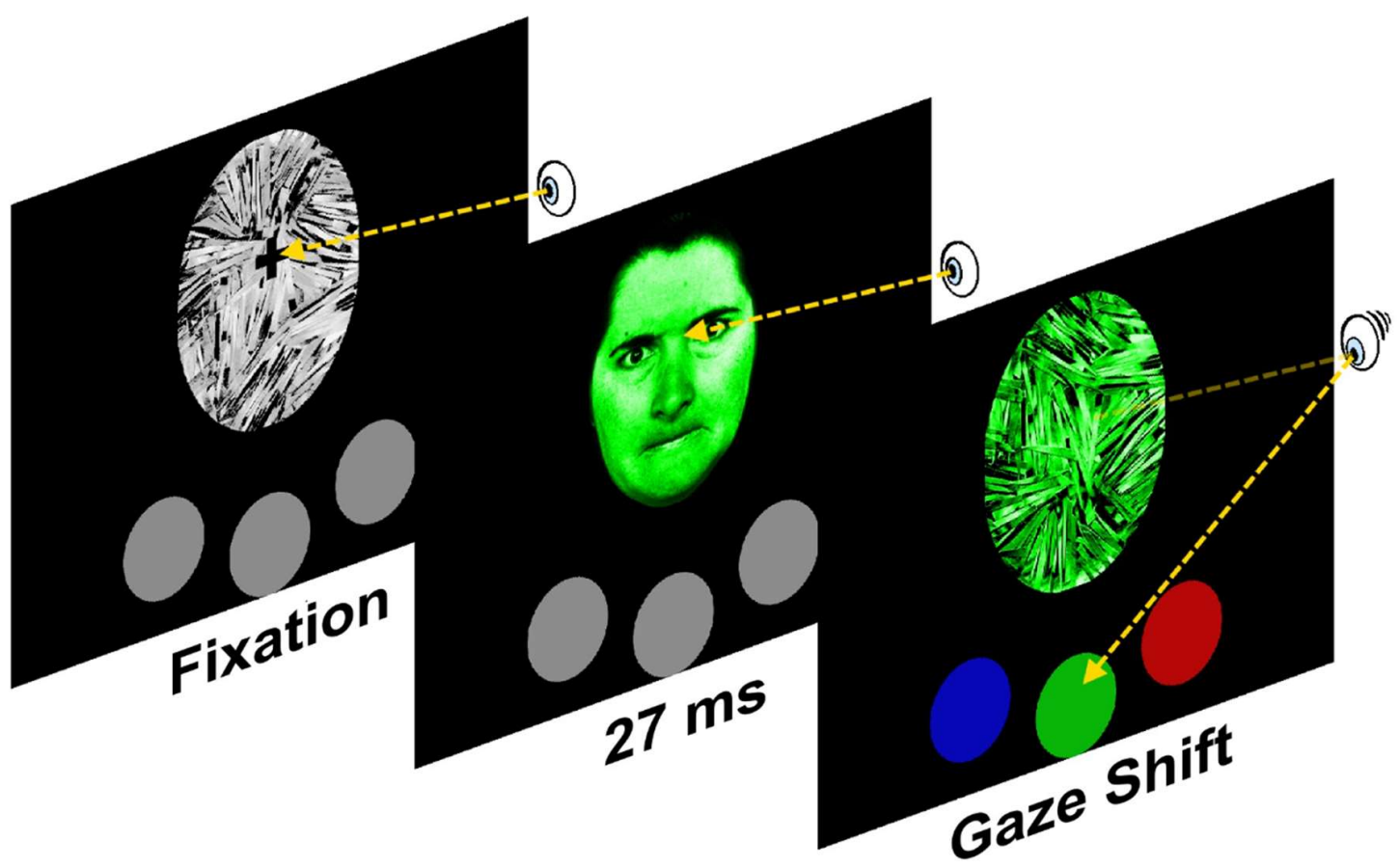

Fig. 1. Example of a trial based stimulus sequence in the gaze-aversion task. The fixation cross was presented for $1000-1500 \mathrm{~ms}$ together with the pre-mask. Stimulus presentation is only initiated if the eye-tracker detects continuous gaze on the fixation cross. The face stimulus in one of three colors (blue, green or red) is presented for $27 \mathrm{~ms}$, followed by the post-mask in the same color. The grey dots presented under the stimulus area change to color (blue, green and red) in randomized order together with appearance of the post-mask. The mask stimulus disappears once the gaze shifts to the color corresponding dot and therewith ends the trial. 


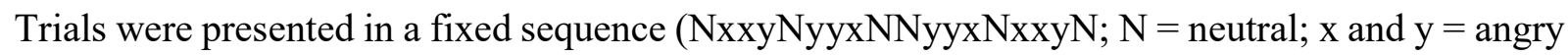
and happy, counterbalanced across the two sessions) repeated 5 times, which allowed to present all possible combinations of successive facial expressions equal amount of times and therefore preventing trial-by-trail emotion carry-over effects. A total of 90 stimuli (10 actors, 3 expressions, 3 colors) were presented during the task, with 10 additional practice trials with neutral facial expressions at the start of the task. Previous research has shown that the difference in gaze-aversion latency for angry and happy faces is a marker for dominance motives (Enter et al., 2016; Terburg et al., 2012, 2011, 2016).

\section{Emotion awareness check}

At the end of the final session, participants were asked to complete a second task in which all 30 faces (10 faces x 3 emotions) were presented again, with backward and forward masking, however this time participants were instructed to identify the emotional valence of the stimulus in a three way forced-choice design. Colors were randomly assigned, however each color was presented 10 times. Emotion awareness was defined as recognition of emotional expressions above chance $(>14$ correct identification; chance level $=10$ correct out of 30; binomial test with one-tailed $\alpha=0.05$ ). Important to note is that the awareness check did not screen for awareness of faces, but awareness of emotional expression specifically.

\section{Touch manipulation}

The touch manipulation was based on a manipulation used in a recently published study by Brummelman, Terburg, Smit, Bögels, \& Bos (2018) investigating the effect of parental touch on social vigilance in children. The standardized procedure looked as follows: Session 2 and 3 both started with preparation of the participant for the different experimental tasks of the study, including placement of electroencephalography (EEG) and electromyography (EMG) electrodes. Next, after starting up the gaze-aversion task, the female experimenter, standing slightly behind the participant on her right, either briefly touched or did not touch the participants shoulder whilst encouraging the participant to start with the experiment. The experimenter subsequently walked out of the room without further interaction. In the touch condition the experimenter placed her left hand on the participant's right shoulder, below the deltoid, for approximately one second (cf. Brummelman et al., 2018). We decided to use a manipulation designed to be short and precise, as well as subtle. Firstly, we were interested in creating a touch scenario that could naturally happen in the laboratory setting, without making the participant uncomfortable. Furthermore, again with the intent of keeping the set up as natural as possible, we aimed for a manipulation that is not consciously perceived by the test subject (cf. Brummelman et al., 2018).

During debrief and manipulation check a total of 11 out of 24 subjects reported that they noticed being touched on the shoulder in the course of the study. 8 of those 11 could report the moment correctly, none of the participants reported the touch as uncomfortable and only 1 person suspected 
it might be part of the experiment at the end of the last session. Awareness about the touch manipulation included as a between subject factor in a separate $2 \times 3$ ANOVA and showed to have no influence on the reported results (all interaction $\mathrm{p}$-values including between subject factor $\mathrm{p}>$ .43 ). We therefore did not exclude any of the here mentioned participants from analysis.

\section{Questionnaires}

To account for individual differences, we asked participants to fill in the Social Touch Questionnaire (STQ) at the end of the final session, a 20 item scale which measures a broad range of attitudes towards social touch (Wilhelm, Kochar, Roth, \& Gross, 2001).

Furthermore, participants were asked to complete the Liebowitz social anxiety scale (LSAS; Safren et al., 1999) questionnaire, which has previously been shown to be predictive for faster gaze-aversion from angry faces in individuals scoring high on the LSAS (Terburg et al., 2016).

\section{Data acquisition and analysis}

Eye movements were recorded with a Tobii Pro TX300 infrared eye tracker (Tobii Technology, Danderyd, Sweden) sampling at $300 \mathrm{~Hz}$, with $0.4^{\circ}$ accuracy. Gaze-aversion latency was defined as the time between the face stimulus onset and the moment the gaze reached the target circle. All latencies that exceeded more than 3 standard deviations of an individual's mean were excluded from the analysis $(2.09 \%$ of all trials).

The effect of social touch on gaze-aversion latencies is tested in two steps. We first evaluate the touch by emotion interaction using a 2 (touch, no-touch) x 3 (angry, happy, neutral) repeated measures ANOVA, with touch and emotion as within subject factors. If successful, we follow-up by testing the main hypothesis that touch will slow-down gaze-aversion from angry, compared to happy, eye-contact using a 2 (touch, no-touch) x 2 (angry, happy) repeated measures ANOVA, and also test for general touch effects on gaze-aversion from neutral eye-contact using a pairedsamples t-test.

To evaluate the influence of STQ and LSAS these analyses are repeated twice, with these questionnaires as respective covariates.

\section{Results}

\section{Main analysis}

As expected social touch affected gaze-aversion from eye-contact in an emotion specific manner (significant touch $\mathrm{x}$ emotion interaction $F(2,46)=3.99, p=.025, \eta_{p}{ }^{2}=.15$, neither a main effect of emotion $F(2,46)=.11, p=.895, \eta_{p}{ }^{2}=.01$, nor a main effect of touch $F(1,23)=.31, p=.583$, $\left.\eta_{p}{ }^{2}=.01\right)$. The averages per condition are presented in Table 1 . In line with our hypothesis social touch induced slower gaze-aversion from angry compared to happy eye-contact $(F(1,23)=5.83$, 
Social touch alters gaze behavior $\mid 8$

$p=.024, \eta_{p}^{2}=.2$; Fig. 2), and also induced marginally slower gaze-aversion from neutral eyecontact $(t(23)=2.01, p=.056)$.

Table 1.

Overview of Average Gaze-Aversion Latencies

\begin{tabular}{|l|l|l|l|}
\hline Condition & Angry & Happy & Neutral \\
\hline \multirow{3}{*}{ Touch } & 412.94 & 402.05 & 417.3 \\
\hline & $95 \%$ CI [388.06, 437.84] & $95 \%$ CI [382.58, 421.51] & $95 \%$ CI [395.46, 439.15] \\
\hline & 404.25 & 412.29 & 400.8 \\
No-touch & $95 \%$ CI [381.6, 426.9] & $95 \%$ CI [387.10, 437.47] & $95 \%$ CI [381.12, 420.5] \\
\hline
\end{tabular}

Means of gaze-aversion latencies in milliseconds for angry, happy and neutral faces in both, touch and no-touch condition. 95\% Confidence Intervals (CI) are indicated.

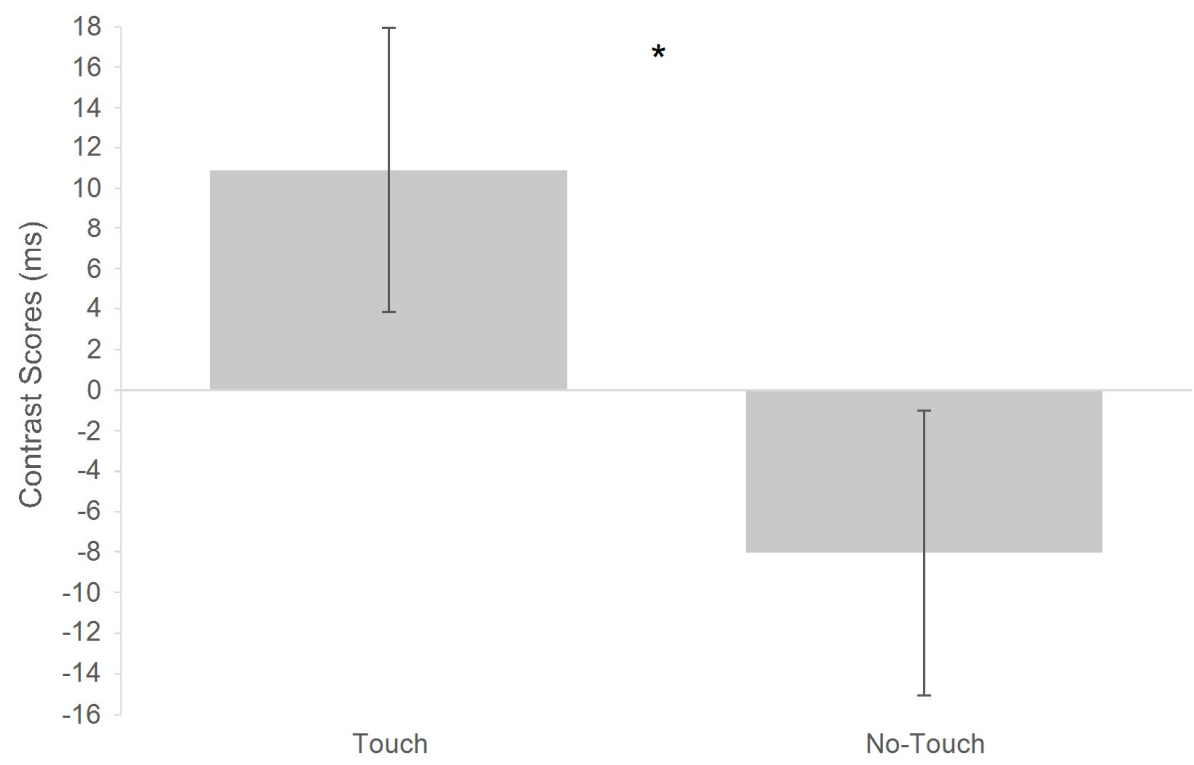

Fig. 2. Contrast scores of angry compared to happy eye-contact. Contrast scores in milliseconds (ms) of angry compared to happy eye-contact, calculated by subtracting mean happy gaze-aversion latencies from mean angry gaze-aversion latencies separately for the touch and notouch condition. Social touch resulted in significantly slower gaze aversion from angry compared to happy eye-contact, with $* \mathrm{p}<.05$. The error bars represent the standard error of the mean. 
Social touch alters gaze behavior $\mid 9$

Post-hoc we checked whether the touch-effect on angry and happy gaze-aversion was independent of the touch-effect on neutral gaze-aversion. For this we first computed for each participant the touch-effect on neutral gaze-aversion (touch minus no-touch) and added these values as covariate to the $2 \times 2$ (touch/no-touch $\mathrm{x}$ angry/happy) repeated measures ANOVA. Results showed that the significant interaction of touch and emotion persisted $\left(F(1,22)=7.22, p=.013, \eta_{p}{ }^{2}=.25\right)$, confirming that the hypothesized touch-effect on gaze-aversion from angry compared to happy eye-contact is independent from the marginal touch-effect on gaze-aversion from neutral eyecontact.

\section{STQ analysis}

To assess whether individual attitudes toward touch predict the effect of touch on gaze-aversion in angry compared to happy faces, we added STQ as a covariate to the $2 \times 2$ (touch/no-touch $\mathrm{x}$ angry/happy) repeated measures ANOVA. The original effect of touch, slowing gaze-aversion from angry compared to happy faces, persisted $\left(F(1,22)=5.79, p=.025, \eta_{p}{ }^{2}=.21\right)$, and the effect seemed to be independent from attitudes around touch (no significant Touch $\mathrm{x}$ Emotion $\mathrm{x}$ STQ interaction $\left.F(1,22)=.84, p=.370, \eta_{p}{ }^{2}=.04\right)$. At the same time, we found a significant interaction of Emotion x STQ $\left(F(1,22)=9.39, p=.006, \eta_{p}{ }^{2}=.3\right)$, suggesting an effect of attitudes around touch on gaze-aversion, independent of the touch manipulation itself. Testing the effect of STQ scores separately on each emotion (touch/no-touch $\mathrm{x}$ angry, touch/no-touch $\mathrm{x}$ happy) confirmed the independence of STQ attitudes from the touch manipulation in both emotions, suggesting rather an effect of attitude towards touch specifically on gaze-aversion from angry compared to happy eye contact. Individuals who are more comfortable with touch (low STQ score) show slower gaze-aversion from angry compared to happy faces compared to individuals who are less comfortable with touch (high STQ score; correlation of angry compared to happy eye contact, touch and no-touch pooled together, correlated with STQ: $r(22)=-.55, \mathrm{p}=.006$ ).

\section{LSAS analysis}

In a third step we included the Liebowitz Social Anxiety Scale (LSAS) as a covariate in the analysis. Social anxiety had no influence on gaze-aversion behavior with or without touch, no near to significant interaction effects with LSAS were found in the $2 \times 2$ (touch/no-touch $\mathrm{x}$ angry/happy) ANOVA (all $\mathrm{p}>.05)$.

\section{Discussion}

In line with our expectations we show that brief social touch results in slower gaze-aversion from subliminally presented angry compared to happy eye-contact, an index of increased dominant behavior in social situations. Previous literature has shown that social touch reduces both psychological and physiological markers of stress and anxiety (Brummelman et al., 2018; Ditzen et al., 2007; Dunbar, 2010) and promotes confident behavior including exploration, readiness to 
engage in difficult challenges and risk taking behavior (Jakubiak \& Feeney, 2016b; Levav \& Argo, 2010). Our study extends those effects found on a psychological and physiological level to implicit and reactive levels of behavior.

In addition to the effect of interest, we found a marginal effect of touch on gaze-aversion from neutral faces, with slower gaze aversion from neutral stimuli after touch. Importantly, the effect was independent from the touch-effect on angry compared to happy faces. A possible interpretation of why touch affects gaze to neutral faces in the same direction as angry faces (cf. Table 1) is that neutral facial stimuli are ambiguous social stimuli and therefore categorized as potentially threatening rather than safe (Lee, Kang, Park, Kim, \& An, 2008).

Analyses of covariance showed that the found main effect is independent from attitudes around touch (STQ) and markers of social anxiety (LSAS). To start with the latter, our results support Brummelman et al. (2018) who showed that LSAS moderates the effect of touch on trustworthiness decisions, but not on implicit reactions to threat as also measured here. Further, interesting for future research is that we found that participants who report to be relatively uncomfortable with social touch are more avoidant in their gaze-aversion behavior in angry compared to happy eye contact. The main finding of interest, the influence of touch on gaze-aversion in angry compared to happy faces, was however not affected by this interaction of STQ with gaze-aversion behavior.

Looking at the possible underpinnings of gaze behavior when conscious processing of the stimuli is prevented, a structure suggested to mediate gaze-aversion is the amygdala. It has been shown to mediate fear and aggression related reflexes in human and non-human primates through its output connections to other subcortical structures, specifically the hypothalamus and brainstem (Davis \& Whalen, 2001; Terburg et al., 2016). Considering the neural underpinnings of social touch in general, the amygdala, amongst other regions, is important to process and code the emotional value of touch and to facilitate the further integration of touch through cortical areas (Boehme, Hauser, Gerling, Heilig, \& Olausson, 2018). Furthermore, touch has been associated to the release of endogenous opioids (Coan et al., 2006; Ellingsen, Leknes, Løseth, Wessberg, \& Olausson, 2016; Morrison, 2016). Opioids have been shown to be involved in the regulation and deactivation of areas responsible for threat and fear processing such as the amygdala (Eippert, Bingel, Schoell, Yacubian, \& Büchel, 2008; Haaker, Yi, Petrovic, \& Olsson, 2017) and therefore are an interesting candidate to consider when looking at the underpinnings of the psychological and behavioral effects of touch.

Last, a clear strength of our study is the touch manipulation. The manipulation is a form of social touch to be found in day to day life and still, partly due to its implicitness, was easy to implement in the experimental context. The manipulation has previously shown to be effective in parent-child context (Brummelman et al., 2018) and now in the context of an unfamiliar other situation. Taken together, the social and implicit aspect of the touch manipulation increase the external validity of the study compared to other approaches, such as using a brush to induce optimal C-tactile afferent stimulating touch. Limitations of the current study are that whilst the findings give us an indication 
about the momentary effects of touch, it does not take long-term effects into account. Another limitation is that this study included female subjects only. Whilst the task has previously been applied in mixed (Hortensius, Van Honk, De Gelder, \& Terburg, 2014; Terburg et al., 2011) as well as female only samples (Terburg et al., 2012, 2016) without showing sex specific differences in task performance, a sex specific effect of touch might exist. Further, we decided for a same-sex experimental set up with a female experimenter implementing the manipulation. It would therefore be important to test for the generalizability of the results in the male population as well as for potential differences in a set up with a same-sex compared to other-sex experimenter in future studies.

\section{Conclusion}

Using an interactive eye-tracking approach, we show that brief social touch by a stranger increases social dominance behavior in female participants, characterized by slower gaze-aversion from subliminally presented angry compared to happy eye-contact (Brummelman et al., 2018; Terburg et al., 2012, 2011, 2016). These results provide evidence that touch adds to psychological resources useful in challenging situations through increased implicit tendencies to engage in social confrontations.

\section{Author contributions}

IM Meier, PA Bos and J van Honk developed the study concept. All authors contributed to the study design. IM Meier led and supervised data collection. IM Meier and D Terburg performed the data analysis. IM Meier drafted the manuscript, D Terburg, PA Bos and J van Honk provided critical revisions. All authors approved the final version of the manuscript for submission.

\section{Declaration of conflict of interest}

The authors declared that they had no conflicts of interest with respect to their authorship or the publication of this article.

\section{Acknowledgements}

We would like to thank Sophie Börner for her help and effort with data collection and testing. 
Social touch alters gaze behavior $\mid 12$

\section{Literature}

Boehme, R., Hauser, S., Gerling, G. J., Heilig, M., \& Olausson, H. (2018). Distinction of selfproduced touch and social touch at cortical and spinal cord levels. https://doi.org/10.1073/pnas.1816278116

Brummelman, E., Terburg, D., Smit, M., Bögels, S. M., \& Bos, P. A. (2018). Parental touch reduces social vigilance in children. Developmental Cognitive Neuroscience, 35(May), 8793. https://doi.org/10.1016/j.dcn.2018.05.002

Burleson, M. H., \& Davis, M. C. (2014). Social Touch and Resilience. In The Resilience Handbook: Approaches to Stress and Trauma (pp. 131-143). Routledge.

Coan, J. a, Schaefer, H. S., \& Davidson, R. J. (2006). Lending a Hand: Social Regulation of the Neural Response to Threat. Psychological Science, 17(12), 1032-1039. https://doi.org/https://doi.org/10.1111/j.1467-9280.2006.01832.x

Davis, M., \& Whalen, P. J. (2001). The amygdala: Vigilance and emotion. Molecular Psychiatry, 6(1), 13-34. https://doi.org/10.1038/sj.mp.4000812

Ditzen, B., Neumann, I. D., Bodenmann, G., von Dawans, B., Turner, R. A., Ehlert, U., \& Heinrichs, M. (2007). Effects of different kinds of couple interaction on cortisol and heart rate responses to stress in women. Psychoneuroendocrinology, 32(5), 565-574. https://doi.org/10.1016/j.psyneuen.2007.03.011

Dunbar, R. I. M. (2010). The social role of touch in humans and primates: Behavioural function and neurobiological mechanisms. Neuroscience and Biobehavioral Reviews, 34(2), 260 268. https://doi.org/10.1016/j.neubiorev.2008.07.001

Eippert, F., Bingel, U., Schoell, E., Yacubian, J., \& Büchel, C. (2008). Blockade of endogenous opioid neurotransmission enhances acquisition of conditioned fear in humans. The Journal of Neuroscience : The Official Journal of the Society for Neuroscience, 28(21), 5465-5472. https://doi.org/10.1523/JNEUROSCI.5336-07.2008

Ellingsen, D. M., Leknes, S., Løseth, G., Wessberg, J., \& Olausson, H. (2016). The Neurobiology Shaping Affective Touch: Expectation, Motivation, and Meaning in the Multisensory Context. Frontiers in Psychology, 6(January), 1-16. https://doi.org/10.3389/fpsyg.2015.01986

Enter, D., Terburg, D., Harrewijn, A., Spinhoven, P., \& Roelofs, K. (2016). Single dose testosterone administration alleviates gaze avoidance in women with Social Anxiety Disorder. Psychoneuroendocrinology, 63, 26-33. https://doi.org/10.1016/j.psyneuen.2015.09.008

Erdfelder, E., Faul, F., \& Buchner, A. (1996). GPOWER : A general power analysis program. Behavior Research Methods, Instruments, \& Computers, 28(1), 1-11. https://doi.org/https://doi.org/10.3758/BF03203630

Faul, F., Erdfelder, E., Lang, A.-G., \& Buchner, A. (2007). G * Power 3 : A flexible statistical power analysis program for the social, behavioral, and biomedical sciences. Behavior Research Methods, 39(2), 175-191. https://doi.org/https://doi.org/10.3758/BF03193146

Haaker, J., Yi, J., Petrovic, P., \& Olsson, A. (2017). Endogenous opioids regulate social threat learning in humans. Nature Communications, 8(May), 1-9. https://doi.org/10.1038/ncomms15495

Hofman, D., Bos, P. a., Schutter, D. J. L. G., \& van Honk, J. (2012). Fairness modulates nonconscious facial mimicry in women. Proceedings of the Royal Society B: Biological Sciences, 279(May), 3535-3539. https://doi.org/10.1098/rspb.2012.0694 
Hofman, Dennis, Terburg, D., van Wielink, L., \& Schutter, D. J. L. G. (2013). Coalescence of dominance motivation and responses to facial anger in resting-state and event-related electrophysiology. NeuroImage, 79, 138-144. https://doi.org/10.1016/j.neuroimage.2013.04.088

Hortensius, R., Van Honk, J., De Gelder, B., \& Terburg, D. (2014). Trait dominance promotes reflexive staring at masked angry body postures. PLOS ONE, 9(12), 1-11. https://doi.org/10.1371/journal.pone.0116232

Jakubiak, B. K., \& Feeney, B. C. (2016a). A Sense of Security: Touch Promotes State Attachment Security. Social Psychological and Personality Science, 7(7), 745-753. https://doi.org/10.1177/1948550616646427

Jakubiak, B. K., \& Feeney, B. C. (2016b). Keep in touch: The effects of imagined touch support on stress and exploration. Journal of Experimental Social Psychology, 65, 59-67. https://doi.org/10.1016/j.jesp.2016.04.001

Lee, E., Kang, J. I., Park, I. H., Kim, J. J., \& An, S. K. (2008). Is a neutral face really evaluated as being emotionally neutral? Psychiatry Research, 157, 77-85. https://doi.org/10.1016/j.psychres.2007.02.005

Levav, J., \& Argo, J. J. (2010). Physical contact and financial risk taking. Psychological Science, 21(6), 804-810. https://doi.org/10.1177/0956797610369493

Mazur, A., \& Booth, A. (1998). Testosterone and social dominance in men. Behavioral and Brain Sciences, 21(3), 353-397.

Morrison, I. (2016). Keep Calm and Cuddle on: Social Touch as a Stress Buffer. Adaptive Human Behavior and Physiology, 344-362. https://doi.org/10.1007/s40750-016-0052-x

Morrison, I., Löken, L. S., \& Olausson, H. (2010). The skin as a social organ. Experimental Brain Research, 204(3), 305-314. https://doi.org/10.1007/s00221-009-2007-y

Safren, S. A., Heimberg, R. G., Horner, K. J., Juster, H. R., Schneier, F. R., \& Liebowitz, M. R. (1999). Factor structure of social fears: The Liebowitz Social Anxiety Scale. Journal of Anxiety Disorders, 13(3), 253-270. https://doi.org/10.1016/S0887-6185(99)00003-1

Terburg, D., Aarts, H., \& Honk, J. van. (2012). Testosterone affects gaze aversion from angry faces outside of conscious awareness. Psychological Science, 23(5), 459-463. https://doi.org/10.1177/0956797611433336

Terburg, D., Hooiveld, N., Aarts, H., Kenemans, J. L., \& van Honk, J. (2011). Eye tracking unconscious face-to-face confrontations: Dominance motives prolong gaze to masked angry faces. Psychological Science, 22(3), 314-319. https://doi.org/10.1177/0956797611398492

Terburg, D., Syal, S., Rosenberger, L. A., Heany, S. J., Stein, D. J., \& Honk, J. van. (2016). Testosterone abolishes implicit subordination in social anxiety. Psychoneuroendocrinology, 72, 205-211. https://doi.org/10.1016/j.psyneuen.2016.07.203

Wilhelm, F. H., Kochar, A. S., Roth, W. T., \& Gross, J. J. (2001). Social anxiety and response to touch: Incongruence between self-evaluative and physiological reactions. Biological Psychology, 58(3), 181-202. https://doi.org/10.1016/S0301-0511(01)00113-2 